\title{
Perfil Lipídico em Crianças Obesas: Efeitos de Dieta Hipocalórica e Atividade Fisica Aeróbica
}

\section{RESUMO}

Dieta hipocalórica e atividade física aeróbia promovem perda de peso e melhora do perfil lipídico de adultos obesos, entretanto pouco se conhece em crianças obesas, sendo este o objetivo do trabalho. Estudamos cinqüenta crianças obesas e dividimos em dois grupos pareados: Grupo D (dieta com 55\% de carboidrato, 30\% de gordura e 15\% de proteína - 1.500 e $1.800 \mathrm{kcal}$ ) e Grupo DE (mesma dieta + atividade física aeróbia 1 hora por dia, três vezes por semana). Após cinco meses, avaliamos: índice de massa corpórea (IMC), triglicerídeos, colesterol total (CT) e frações. Nenhuma modificação foi observada nos triglicerídeos, CT e lipoproteína de baixa-densidade colesterol (LDL-C) em ambos os grupos. Houve, porém, aumento da lipoproteína de altadensidade colesterol (HDL-C) apenas no grupo DE (+10,3\%, $p<0,01)$. Selecionando pacientes com CT > $170 \mathrm{mg} / \mathrm{dL}, \mathrm{LDL}-\mathrm{C}>110 \mathrm{mg} / \mathrm{dL}$ e HDL$\mathrm{C}<35 \mathrm{mg} / \mathrm{dL}$, observou-se redução semelhante do CT nos dois grupos $(-6,0 \% \times-6,0 \% ; p=n s)$, assim como da LDL-C de ambos $(-14,2 \% \times-13,5 \%$; $\mathrm{p}=\mathrm{ns})$, e um acréscimo da HDL-C apenas no grupo DE $(+10,0 \% ; \mathrm{p}<0,05)$. Conclusões: 1) Dieta hipocalórica (DH) e atividade física aeróbia promovem aumento da HDL-C, independente do valor basal, em crianças obesas quando comparado à $\mathrm{DH}$ isoladamente; 2) $\mathrm{DH}$ isoladamente ou associada a exercício aeróbio reduz CT e LDL-C, quando estes estão em níveis acima do valor normal, em crianças obesas. (Arq Bras Endocrinol Metab 2006;50/3:499-504)

Descritores: Criança; Obesidade; Lipoproteínas; Exercício; Dieta artigo original

Evika B. Parente

Isabel Guazzelli

Maurício M. Ribeiro

Alexandre G. Silva

Alfredo Halpern

Sandra M. Villares

Grupo de Obesidade e Síndrome Metabólica da Disciplina de Endocrinologia - Departamento de Clínica Médica, Hospital das Clinicas da Faculdade de Medicina da USP, São Paulo, SP.

\section{ABSTRACT}

\section{Obese Children Lipid Profile: Effects of Hypocaloric Diet and Aerobic Physical Exercise.}

Diet and exercise help improve obese adults' lipid profile. However, their effect on obese children, the aim of the present study, is poorly known. Fifty obese children were studied into 2 paired groups: Group D (1,500 - 1,800 kcal diet: $55 \%$ carbohydrate, $30 \%$ fat, $15 \%$ protein), and Group DE (same diet + aerobic physical activity 1 hour/day 3 times a week). After 5 months $\mathrm{BMl}$, triglycerides, total cholesterol (TC) and fractions were assessed. No change in triglycerides, TC and low-density lipoprotein cholesterol (LDL-C) levels were reported in both groups. However, high-density lipoprotein cholesterol (HDL-C) increased (+10.3\%; $\mathrm{P}<0.01)$ only in DE Group. Screening patients with TC $>170 \mathrm{mg} / \mathrm{dL}, \mathrm{LDL}-\mathrm{C}>110 \mathrm{mg} / \mathrm{dL}$ and $\mathrm{HDL}-\mathrm{C}<35$ $\mathrm{mg} / \mathrm{dL}$ we had: similar reduction for TC in both groups (-6.0\% x -6.0\%; $\mathrm{p}=\mathrm{ns}$ ), LDL-C reduction in both groups $(-14.2 \% \times-13.5 \% ; \mathrm{P}=\mathrm{ns})$, and $\mathrm{HDL}-\mathrm{C}$ increase only in DE Group (+10.0\%; $p<0.05)$. Conclusions: 1) Hypocaloric diet $(H D)+$ exercise, rather than diet only, increase obese children's HDLC levels irrespective of baseline levels; 2) HD only and HD + exercise lead to TC and LDL-C reduction in obese children with TC and LDL-C above normal values. (Arq Bras Endocrinol Metab 2006;50/3:499-504)

Keywords: Child; Obesity; Lipoproteins; Exercise; Diet

Recebido em 06/07/04

Revisado em 20/07/04 e 05/04/05

Aceito em 22/02/06 
A PREVALÊNCIA DE OBESIDADE INFANTIL tem aumentado dramaticamente nas duas últimas décadas (1), assim como a hipercolesterolemia, uma das complicações do excesso de peso (2). Alguns trabalhos mostram alterações de risco para doença aterosclerótica e calcificações na aorta e artérias coronárias em crianças obesas $(3,4)$. Diante da preocupação de esta população já apresentar fatores de risco cardiovasculares e da possibilidade de tornarem-se adultos dislipidêmicos (2,5-7), surgem trabalhos na tentativa de intervir precocemente com dietas hipocolesterolêmicas e atividade física (8-12).

Os dados da literatura sobre a influência do exercício aeróbio no perfil lipídico de crianças obesas ainda são escassos $(13,14)$. Existem estudos mostrando melhora do perfil lipídico com atividade física aeróbia (15-17), enquanto outros não demonstram esta alteração $(14,18,19)$, e poucos trabalhos foram realizados em crianças obesas (20). Em revisão sobre os guidelines de atividade física para crianças e adolescentes, viu-se que vários dados não têm embasamento científico consistente e as metodologias são questionáveis $(21)$.

Neste estudo, procuramos avaliar o efeito da atividade física aeróbia associado a dieta hipocalórica balanceada sobre o perfil lipídico (em particular sobre as frações do colesterol), visto ser este um importante fator de risco cardiovascular e haver pouca informação na literatura sobre este tipo de intervenção em crianças obesas, principalmente em nossa população.

\section{MATERIAL E MÉTODOS}

Foram estudadas 50 crianças obesas ( 25 do sexo masculino) selecionadas da população da cidade de São Paulo, que procurou espontaneamente o atendimento do ambulatório de Obesidade Infantil do Hospital das Clínicas no ano de 2002. Critérios de inclusão: diagnóstico de obesidade, caracterizado por índice de massa corporal $\left(\mathrm{IMC}=\right.$ peso/altura $\left.{ }^{2}\right)$ acima do percentil noventa e cinco, ajustado para idade e sexo, segundo curva de IMC do Centers for Diseases Control and Prevention (CDC) Growth Charts (www.cdc.gov/growthcharts/), idade entre 8 e 14 anos. Critérios de exclusão: doença psiquiátrica, doenças endocrinológicas (ex.: hipotireoidismo, síndrome de Cushing, pan-hipopituitarismo) e quaisquer doenças crônicas, uso de qualquer medicação que pudesse interferir no peso do paciente ou nas lipoproteínas (glicocorticóide, diuréticos, antidepressivos tricíclicos ou inibidores da recaptação de sero- tonina, estatinas, fibratos, inibidores da enzima conversora de angiotensina) ou estar participando de algum tipo de tratamento para perda de peso.

As crianças foram divididas em 2 grupos de forma randômica (26 no grupo dieta: 20 do sexo feminino; 24 no grupo dieta e exercício: 5 do sexo feminino). Os dois grupos foram pareadas de forma aleatória para idade, IMC, massa magra, massa gorda, CT, LDL-colesterol (LDL-C) e HDL-colesterol (HDL-C) (tabela 1). O diagnóstico de dislipidemia seguiu as normas da American Heart Association (22). O grupo de 26 crianças submetido à dieta (Grupo D) de 1.500 a $1.800 \mathrm{kcal}$ não fez atividade física, enquanto o outro grupo de 24 crianças (Grupo DE) recebeu a mesma dieta, porém com atividade física programada. A dieta era balanceada com 55\% de carboidrato, $30 \%$ de gordura e $15 \%$ de proteína, e a atividade física constituía-se de uma hora de exercício aeróbio (caminhada, corrida, bicicleta e jogos lúdicos), 3 vezes por semana, com freqüência cardíaca máxima entre o ponto de compensação residual (ponto a partir do qual utiliza-se gordura como fonte de energia de forma aeróbia) e limiar anaeróbico (ponto a partir do qual realiza-se atividade anaeróbia). Todas as crianças foram submetidas a um teste ergoespirométrico (aparelho Sensor Medics) para cálculo do limiar anaeróbico e o ponto de compensação residual. Avaliou-se IMC, massa magra e massa gorda, triglicerídeos, CT e frações no início e ao fim de cinco meses de seguimento mensal, de todas as crianças, com médico, nutricionista e psicólogo. A composição corporal foi aferida pelo método de bioimpedância (aparelho Bia-Quantum 1010, RJL Systems) no período da manhã, após jejum de 12 horas. Os lipídeos foram dosados no período da manhã após jejum de 12 horas, no laboratório de investigação médica número dez (LIM 10) da Faculdade de Medicina da Universidade de São Paulo, através do método enzimático colorimétrico, utilizando os seguintes kits comerciais: colesterol, da Boehringer-Mannhein (Argentina) e triglicerídeos, da Merkotest (Alemanha), para aparelho automatizado Cobas Mira.

Análise estatística realizada através do teste ANOVA, considerando significativo $\mathrm{p}<0,05$.

O estudo foi aprovado pelo Comitê de Ética Médica do Hospital das Clínicas da Faculdade de Medicina da Universidade de São Paulo.

\section{RESULTADOS}

Ambos os grupos eram pareados para idade, IMC, massa magra, massa gorda, CT, LDL-C e HDL-C (tabela 1). 
Tabela 1. Características iniciais de ambos os grupos de crianças.

\begin{tabular}{lccc}
\hline & $\begin{array}{c}\text { Grupo Dieta } \\
\text { (6 M / 20 F) }\end{array}$ & $\begin{array}{c}\text { Grupo Dieta e Exercício } \\
\text { (19 M / 5 F) }\end{array}$ & p $^{*}$ \\
\hline n (total) & 26 & 24 & $\mathrm{NS}$ \\
Idade (anos) & $10,4 \pm 1,5$ & $10,4 \pm 1,4$ & $\mathrm{NS}$ \\
Peso (kg) & $65,4 \pm 17,6$ & $62,5 \pm 11,5$ & $\mathrm{NS}$ \\
IMC (kg/m²) & $30,2 \pm 5,5$ & $29,1 \pm 3,9$ & $\mathrm{NS}$ \\
Massa gorda (kg) & $23,1 \pm 9,2$ & $22,5 \pm 6,7$ & $\mathrm{NS}$ \\
Massa magra (kg) & $38,6 \pm 10,4$ & $38,7 \pm 7,3$ & $\mathrm{NS}$ \\
\% massa gorda & $36,5 \pm 5,7$ & $38,2 \pm 5,9$ & $\mathrm{NS}$ \\
CT (mg/dL) & $182,2 \pm 34,2$ & $169,5 \pm 31,0$ & $\mathrm{NS}$ \\
HDL-C (mg/dL) & $39,5 \pm 7,3$ & $40,3 \pm 6,4$ & $\mathrm{NS}$ \\
LDL-C (mg/dL) & $119,8 \pm 28,8$ & $112,3 \pm 25,7$ & $\mathrm{NS}$ \\
VLDL-C (mg/dL) & $22,9 \pm 8,5$ & $16,6 \pm 7,0$ & $<0,05$ \\
Triglicerídeos (mg/dL) & $117,0 \pm 46,7$ & $83,6 \pm 35,0$ & $<0,05$ \\
\hline * teste ANOVA, valores expressos em média \pm desvio-padrão. & \\
CT= colesterol total; LDL-C= LDL colesterol; HDL-C= HDL colesterol; VLDL-C= VLDL \\
colesterol.
\end{tabular}

Tabela 2. IMC e composição corporal dos pacientes do grupo D e do grupo DE, inicial e após 5 meses.

\begin{tabular}{|c|c|c|c|}
\hline & \multicolumn{2}{|c|}{ Grupo D $(n=26)$} & \multirow{2}{*}{$\frac{D \text { inicial } x \text { final }}{p}$} \\
\hline & inicial & final & \\
\hline $\begin{array}{l}\text { IMC }\left(\mathrm{kg} / \mathrm{m}^{2}\right) \\
\text { Massa gorda }(\mathrm{kg}) \\
\text { Massa magra }(\mathrm{kg}) \\
\text { \% Massa gorda }\end{array}$ & $\begin{array}{c}30,2 \pm 5,5 \\
23,1 \pm 9,2 \\
38,6 \pm 10,3 \\
36,5 \pm 5,7\end{array}$ & $\begin{array}{c}28,3 \pm 5,0 \\
21,5 \pm 8,3 \\
38,7 \pm 10,5 \\
35,1 \pm 4,8\end{array}$ & $\begin{array}{c}<0,001 \\
\text { NS } \\
\text { NS } \\
\text { NS }\end{array}$ \\
\hline \multirow{2}{*}{ \% Massa gorda } & \multicolumn{2}{|c|}{ Grupo DE $(n=24)$} & $D E$ inicial $x$ final \\
\hline & inicial & final & $\mathbf{p}$ \\
\hline $\begin{array}{l}\text { IMC }\left(\mathrm{kg} / \mathrm{m}^{2}\right) \\
\text { Massa gorda }(\mathrm{kg}) \\
\text { Massa magra }(\mathrm{kg}) \\
\% \text { Massa gorda }\end{array}$ & $\begin{array}{l}29,1 \pm 3,9 \\
22,5 \pm 6,7 \\
38,9 \pm 7,3 \\
38,2 \pm 5,9\end{array}$ & $\begin{array}{c}25,5 \pm 3,5^{\star *} \\
16,4 \pm 6,0^{\star *} \\
39,0 \pm 7,6 \\
29,5 \pm 6,7^{\star}\end{array}$ & $\begin{aligned} &< 0,001 \\
&<0,001 \\
& N S \\
&< 0,001\end{aligned}$ \\
\hline
\end{tabular}

* $p<0,01$ comparado à mesma variável do grupo $D$ final.

** $p<0,001$ comparado à mesma variável do grupo $D$ final.

Valores expressos em média \pm desvio-padrão, teste ANOVA.

Ocorreu redução importante do IMC em ambos os grupos, sendo superior no grupo que realizou atividade física $(-13,5 \% \times-6,2 \% ; \mathrm{p}<0,001)$, e houve diminuição da massa gorda apenas no grupo DE $(-27 \% ; \mathrm{p}<0,01)$ com manutenção da massa magra (tabela 2).

Houve incremento do HDL-C $(+10,4 \%$; $<<$ $0,01)$ apenas no grupo $\mathrm{DE}$, porém não foi vista modificação significativa nos níveis de triglicerídeos, CT e LDL-C dos 2 grupos (tabela 3 ). Entretanto, ao sele- cionarmos apenas pacientes com colesterol alterado: $\mathrm{CT}>170 \mathrm{mg} / \mathrm{dL}, \mathrm{LDL}-\mathrm{C}>110 \mathrm{mg} / \mathrm{dL}$ e HDL-C < $35 \mathrm{mg} / \mathrm{dL}$, observamos diminuição semelhante do CT $(-9,9 \% \mathrm{x}-12,6 \% ; \mathrm{p}=\mathrm{ns})$ e do LDL-C $(-15,9 \% \mathrm{x}$ $-14,3 \% ; \mathrm{p}=\mathrm{ns}$ ) em ambos os grupos (tabela 4$)$. Houve elevação significativa do HDL-C apenas no grupo DE $(+32,8 \% ; \mathrm{p}<0,05)$ (tabela 4$)$. Todas as crianças do grupo DE com HDL-C $<35 \mathrm{mg} / \mathrm{dL}$ ficaram com média de HDL-C superior a $35 \mathrm{mg} / \mathrm{dL}$ após 5 meses de exercício. 
Tabela 3. Perfil lipídico dos pacientes do grupo D e do grupo DE, inicial e após 5 meses.

\begin{tabular}{|c|c|c|c|}
\hline & \multicolumn{2}{|c|}{ Grupo D $(n=26)$} & \multirow{2}{*}{$\begin{array}{c}\text { inicial } x \text { final } \\
p\end{array}$} \\
\hline & inicial & final & \\
\hline $\begin{array}{l}\mathrm{CT}(\mathrm{mg} / \mathrm{dL}) \\
\mathrm{HDL}-\mathrm{C}(\mathrm{mg} / \mathrm{dL}) \\
\mathrm{LDL}-\mathrm{C}(\mathrm{mg} / \mathrm{dL}) \\
\text { VLDL-C }(\mathrm{mg} / \mathrm{dL}) \\
\text { Triglicerídeos }(\mathrm{mg} / \mathrm{dL})\end{array}$ & $\begin{aligned} 182,2 & \pm 34,2 \\
39,5 & \pm 7,3 \\
119,8 & \pm 28,8 \\
22,9 & \pm 8,5 \\
117,0 & \pm 46,7\end{aligned}$ & $\begin{array}{c}170,8 \pm 28,8 \\
38,5 \pm 7,4 \\
106,7 \pm 26,9 \\
25,5 \pm 13,0 \\
127,0 \pm 65,2\end{array}$ & $\begin{array}{l}\text { NS } \\
\text { NS } \\
\text { NS } \\
\text { NS } \\
\text { NS }\end{array}$ \\
\hline \multirow{2}{*}{ Triglicerídeos (mg/dL) } & \multicolumn{2}{|c|}{ Grupo DE $(n=24)$} & inicial $x$ final \\
\hline & inicial & final & $p$ \\
\hline $\begin{array}{l}\text { CT }(\mathrm{mg} / \mathrm{dL}) \\
\mathrm{HDL}-\mathrm{C}(\mathrm{mg} / \mathrm{dL}) \\
\text { LDL-C (mg/dL) } \\
\text { VLDL-C (mg/dL) } \\
\text { Triglicerídeos (mg/dL) }\end{array}$ & $\begin{array}{c}169,5 \pm 31,0 \\
40,3 \pm 6,4 \\
112,3 \pm 25,7 \\
16,6 \pm 7,0^{*} \\
83,6 \pm 35,0^{*}\end{array}$ & $\begin{array}{c}159,0 \pm 27,2 \\
44,5 \pm 7,4^{\star \star *} \\
97,8 \pm 22,4 \\
16,7 \pm 8,0^{\star *} \\
83,87 \pm 39,7^{\star *}\end{array}$ & $\begin{array}{l}\text { NS } \\
<0,01 \\
\text { NS } \\
\text { NS } \\
\text { NS }\end{array}$ \\
\hline
\end{tabular}

Tabela 4. Perfil lipídico dos pacientes com CT > $170 \mathrm{mg} / \mathrm{dL}, \mathrm{LDL}-\mathrm{C}>110 \mathrm{mg} / \mathrm{dL}, \mathrm{HDL}-\mathrm{C}<35$ $\mathrm{mg} / \mathrm{dL}$, antes e após intervenção.

\begin{tabular}{|c|c|c|c|}
\hline & \multicolumn{2}{|c|}{ Grupo D } & \multirow{2}{*}{$\frac{\text { inicial } x \text { final } D}{p}$} \\
\hline & inicial & final & \\
\hline \multirow[t]{3}{*}{$\begin{array}{l}\text { CT }(\mathrm{mg} / \mathrm{dL})(\mathrm{n}=17) \\
\mathrm{HDL}-\mathrm{C}(\mathrm{mg} / \mathrm{dL})(\mathrm{n}=6) \\
\mathrm{LDL}-\mathrm{C}(\mathrm{mg} / \mathrm{dL})(\mathrm{n}=15)\end{array}$} & $\begin{array}{c}202,0 \pm 22,0 \\
30,0 \pm 4,2 \\
139,7 \pm 15,7\end{array}$ & $\begin{array}{c}182,0 \pm 28,3 \\
36,5 \pm 6,3 \\
117,4 \pm 28,6\end{array}$ & $\begin{array}{l}<0,05 \\
\quad N S \\
<0,01\end{array}$ \\
\hline & \multicolumn{2}{|c|}{ Grupo DE } & inicial $x$ final $D E$ \\
\hline & inicial & final & $\mathrm{p}$ \\
\hline $\begin{array}{l}\text { CT }(\mathrm{mg} / \mathrm{dL})(\mathrm{n}=11) \\
\mathrm{HDL}-\mathrm{C}(\mathrm{mg} / \mathrm{dL})(\mathrm{n}=5) \\
\mathrm{LDL}-\mathrm{C}(\mathrm{mg} / \mathrm{dL})(\mathrm{n}=11)\end{array}$ & $\begin{array}{c}197,0 \pm 20,0 \\
32,0 \pm 2,8 \\
153,0 \pm 12,7\end{array}$ & $\begin{array}{c}172,0 \pm 28,7 \\
42,5 \pm 4,9^{*} \\
131,0 \pm 18,4\end{array}$ & $\begin{array}{l}<0,05 \\
<0,05 \\
<0,01\end{array}$ \\
\hline
\end{tabular}

\section{DISCUSSÃO}

Desde que obesidade e hipercolesterolemia foram consideradas como fatores de risco cardiovascular, estudos surgiram na tentativa de modificar estes fatores já na infância, e a mudança do estilo de vida com dieta e exercício tornou-se uma arma importante (8-23).

Após cinco meses de intervenção não percebemos alteração nos níveis de CT, LDL-C, triglicerídeos ou lipoproteína de muito baixa-densidade colesterol (VLDL-C), avaliando todas as crianças de ambos os grupos. Provavelmente este resultado deve-se ao fato de muitas crianças apresentarem níveis de CT e frações dentro do limite da normalidade (22). Vale também ressaltar que a média inicial de triglicerídeos não era elevada (tabela 1), sugerindo que hipertrigliceridemia não é uma alteração metabólica comum na obesidade infantil, como no obeso adulto. Apesar de perderem peso, as crianças não diminuíram os níveis séricos de triglicerídeos, fato que pode ser explicado possivelmente devido à grande quantidade de gordura que estas crianças ingeriam em seu cotidiano (em média $50 \%$ do total de kcal da dieta), e com a introdução da dieta hipocalórica balanceada houve aumento da proporção de carboidratos em relação à quantidade de gordura da dieta. Ao avaliarmos, em ambos os grupos, apenas as crianças com CT e LDL-C acima dos valores normais, observamos que esta redução da quantidade de gordura total diária da dieta foi relevante, tornando significativa a diminuição do CT e LDL-C neste grupo 
específico. Nossos dados são compatíveis com outros estudos da literatura, em que se avaliou apenas a dieta em crianças obesas $(9,10)$. Em recente estudo observacional, foi demonstrada queda do CT, LDL-C em um grupo de crianças submetidas a dieta e exercício, mas não houve aumento significativo do HDL-C, apenas uma tendência (24). Talvez este resultado seja diferente do nosso porque, como vimos, a atividade física é o fator relevante no aumento do HDL-C. No trabalho da literatura, o grupo dieta e exercício foi comparado ao grupo controle sem dieta e sem exercício, desta forma não foi levada em consideração a variável exercício isoladamente, como fizemos. Assim, não sabemos o quanto a dieta ou o exercício no grupo tratado contribuiu para melhorar o perfil lipídico. Se poucas crianças fizeram atividade física, teremos um grupo onde a dieta é o fator mais importante e, de acordo com nossos dados, a dieta faz reduzir CT e LDL-C, mas não aumenta HDL-C.

Em nosso estudo, a melhora do perfil lipídico no grupo dieta foi restrita àqueles com CT e LDL-C acima do valor normal, indicando ser esta a população de maior benefício para este tipo de intervenção.

A dieta hipocalórica balanceada isoladamente não causou nenhuma alteração significativa no HDL$\mathrm{C}$ no grupo $\mathrm{D}$, enquanto no grupo $\mathrm{DE}$ observamos incremento significativo do HDL-C, não somente naqueles pacientes com valores inferiores a $35 \mathrm{mg} / \mathrm{dL}$ inicialmente, como também naqueles com HDL-C normal. Como não houve modificação nos níveis de triglicerídeos, é possível que esta elevação do HDL-C se deva ao efeito do exercício nesta fração do colesterol $(13,15,16)$. Quanto às demais frações de colesterol no grupo DE, a diminuição do CT e LDL-C foi semelhante ao grupo $\mathrm{D}$ e restrita aos pacientes com níveis acima do limite da normalidade.

Ao término do estudo, reavaliamos o IMC e a composição corporal de ambos os grupos e os dois obtiveram redução do IMC, sendo maior no grupo DE. Além de constatarmos maior redução do IMC, a atividade física proporcionou melhora da composição corporal destas crianças, diminuindo massa gorda e preservando massa magra (25). Tal fato corrobora a idéia de que atividade física não somente contribui para diminuição do peso corporal, mas favorece o equilíbrio saudável entre massa gorda e massa magra.

Dieta hipocalórica isolada e dieta hipocalórica associada à atividade física aeróbia levam à diminuição do CT e LDL-C em crianças obesas dislipidêmicas. A dieta hipocalórica com atividade física (e não apenas dieta) eleva níveis de HDL-C em crianças obesas independentemente do valor basal, e melhora a compo- sição corporal destas crianças diminuindo massa gorda. Tais modificações induzidas pela atividade física aeróbia associada à dieta em crianças obesas, se utilizadas cronicamente, possivelmente contribuirão para redução de eventos cardiovasculares na idade adulta.

\section{REFERÊNCIAS}

1. Centers for Diseases Control and Prevention Update: prevalence of overweight among children, adolescents and adults-United States, 1988-1994. JAMA 1997; 277:1111.

2. Bao W, Srinivasan SR, Wattigney WA, Bao W, Berenson GS. Usefulness of childhood low-density lipoprotein cholesterol level in predicting adult dyslipidemia and other cardiovascular risks. The Bogalusa Heart Study. Arch Intern Med 1996; 156:1315-20.

3. Mahoney LT, Burns TL, Stanford W, Thompson BH, Witt JD, Rost $C A$, et al. Coronary risk factors measured in childhood and young adult life are associated with coronary artery calcification in young adults: The Muscatine Study. J Am Coll Cardiol 1996;27:277-84.

4. Berenson GS, Srinivasan SR, Bao W, Newman III WP, Tracy RE, Wattigney WA. Association between multiple cardiovascular risk factors and atherosclerosis in children and young adults: The Bogalusa Heart Study. N Eng J Med 1998:338:1650-6.

5. Lauer RM, Lee J, Clarke WR. Factors affecting the relationship between childhood and adult cholesterol level: The Muscatine Study. Pediatrics 1988;82:309-18.

6. Hickman TB, Briefel RR, Carroll MD, Rifkind BM, Cleeman $\mathrm{JI}$, Maurer KR, et al. Distribution and trends of serum lipids among United States children and adolescents ages 419 Years: data from the Third National Health and Nutrition Examination Survey. Prev Med 1998:27:879-90.

7. Lauer RM, Lee J, Clarke WR. Predicting adult cholesterol levels from measurements in childhood and adolescence: The Muscatine Study. Bull N Y Acad Med 1989;65:1127-42.

8. Obarzanek E, Kimm SY, Barton BA, Van Horn LL, Kwiterovich PO Jr, Simons-Morton DG, et al. Long-term safety and efficacy of a cholesterol-lowering diet in children with elevated low-density lipoprotein cholesterol: seven-year results of the Dietary Intervention Study in Children (DISC). Pediatrics 2001; 107:256-64.

9. Endo $H$, Takagi $Y$, Nozue $T$, Kuwahata $K$, Uemasu $F$, Kobayashi A. Beneficial effects of dietary intervention on serum lipid and apolipoprotein levels in obese children. Am J Dis Child 1992; 146(3):303-5.

10. Muziotti FMC, Román VB, Rodríguez Ml. (Effects of nutritional intervention in anthropometrics variables, ingestion and plasma lipids and lipoproteins concentrations in dyslipidemic children) (Spanish). Arch Latinoam Nutr 2001:51:132-44.

11. Freedman DS, Dietz WH, Srinivasan SR, Berenson GS. The relation of overweight to cardiovascular risk factors among children and adolescents: The Bogalusa Heart Study. Pediatrics 1999; 103:1175-82. 
12. Williams CL, Hayman LL, Daniels SR, Robinson TN, Steinberger J, Paridon S, et al. Cardiovascular health in childhood. A statement for health professionals from the committee on atherosclerosis, hypertension, and obesity in the young (AHOY) of the council on cardiovascular disease in the young, American Heart Association. Circulation 2002; 106: 143-60.

13. Tolfrey K, Jones AM, Campbell IG. The effect of aerobic exercise training on the lipid-lipoprotein profile of children and adolescents. Sports Med 2000;29(2):99-112.

14. Tolfrey K, Jones AM, Campbell IG. Lipid-lipoproteins in children: an exercise dose-response study. Med Sci Sports Exerc 2004;36(3):418-27.

15. Eisenmann JC, Katzmarzyk PT, Perusse L, Bouchard C, Malina RM. Estimated daily energy expenditure and blood lipids in adolescents: the Québec Family Study. J Adolesc Health 2003;33(3): 147-53.

16. Saakslahti A, Numminen P, Varstala V, Helenius H, Tammi $A$, Viikari $J$, et al. Physical activity as a preventive measure for coronary heart disease risk factors in early childhood. Scand J Med Sci Sports 2004; 14(3): 143-9.

17. Sarria Chueca A, Martin Nasarre de Letosa MT, Lomba Garcia B, Moreno Aznar LA, Lazaro Almarza A, Bueno Sanchez M. (Lipoprotein profile determinants in children and adolescents from a lipid consultation clinic. The impact of diet, body composition and physical activity) (Spanish). An Esp Pediatr 1997:47(4):357-62

18. Linder $\mathrm{CW}$, DuRant RH, Mahoney OM. The effect of physical conditioning on serum lipids and lipoproteins in white male adolescents. Med Sci Sports Exerc 1983; 15(3):232-6.

19. Suter E, Hawes MR. Relationship of physical activity, body fat, diet, and blood lipid profile in youths 10-15 yr. Med Sci Sports Exerc 1993;25(6):748-54.
20. Sasaki J, Shindo M, Tanaka H, Ando M, Arakawa K. A long-term aerobic exercise program decreases the obesity index and increases the high-density lipoprotein cholesterol concentration in obese children. Int J Obes Relat Metab Disord 1987; 1 (4):339-45.

21. Twisk JWR. Physical activity guidelines for children and adolescents: A critical review. Sports Med 2001;31:617-27.

22. Kavey REW, Daniels SR, Lauer RM, Atkins DL, Hayman LL, Taubert K. American Heart Association Guidelines for primary prevention of atherosclerotic cardiovascular disease beginning in childhood. Circulation 2003; 107:1562-6.

23. Becque MD, Katch VL, Rocchini AP, Marks CR, Moorehead $C$. Coronary risk incidence of obese adolescents: reduction by exercise plus diet intervention. Pediatrics 1988;81(5):605-12.

24. Korsten-Reck U, Kromeyer-Hauschlid K, Wolfarth B, Dickhuth $\mathrm{HH}$, Berg A. Freiburg Intervention Trial for Obese Children (FITOC): results of a clinical observation study. Int J Obes Relat Metab Disord 2005;29(4):356-61

25. Sothern MS, Hunter S, Suskind RM, Brown R, Udall JN, Blecker U. Motivating the obese child to move: the role of structured exercise in pediatric weight management. South Med J 1999:92:577-83.

\section{Endereço para correspondência:}

Érika Bezerra Parente

Av. Higienópolis 968, ap. 71B

01238-000 São Paulo, SP 